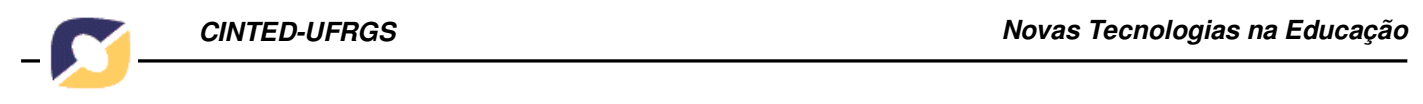

\title{
EXPERIÊNCIAS NO APRENDIZADO DE ALGORITMOS UTILIZANDO UM AMBIENTE DE APRENDIZAGEM
}

\author{
Fabrício Viero de Araújo \\ Faculdade Dom Alberto - Santa Cruz do Sul - RS - Brasil, vieros@terra.com.br \\ Gilse A. Morgental Falkembach \\ Universidade Luterana do Brasil ULBRA - Santa Maria - RS - Brasil, \\ gilsemf@terra.com.br
}

\begin{abstract}
This work reference an adapted learning environment, totally created in Free Software, with interactive multimedia resources, added of the support modules to the student's learning and the teacher. It contains examples with animations that aid in the visualization of the internal operations during the execution of a program. The result in versions previous of the environment shows that the learning of the necessary content to the development of an algorithm was excelled, increasing in the students the capacity to abstract and to formalize.
\end{abstract}

Resumo: Este trabalho referencia um ambiente de aprendizagem adaptado, criado totalmente em Software Livre, com os recursos da multimídia interativa, acrescido dos módulos de suporte ao aprendizado do aluno e ao professor. Contém exemplos com animações que auxiliam na visualização das operações internas durante a execução de um programa. O resultado em versões anteriores do ambiente mostram que a aprendizagem do conteúdo necessário ao desenvolvimento de um algoritmo foi potencializada, aumentando nos alunos a capacidade de abstrair e formalizar.

Keywords: Hypermedia, e-learning, Algorithms

\section{Introdução}

Este artigo se refere às experiências do uso da Estratégia Ascendente de Resolução de Problemas, em um ambiente de aprendizagem adaptado, criado com os recursos da multimídia interativa, contendo a teoria referente à disciplina de Algoritmos e exemplos com animações que auxiliam na visualização das operações internas durante a execução de um programa.

Considerando as dificuldades encontradas pelos alunos dos cursos da área de Ciências Exatas no aprendizado de Algoritmos considerando que esse conteúdo é de fundamental importância para os cursos da área de Informática; considerando também que cada aluno tem o seu ritmo de trabalho e que não é possível ao professor adequar-se às necessidades de cada aluno, esses fatores justificaram o desenvolvimento do Ambiente de Aprendizagem Adaptado para Algoritmos, o A4, pois através de inovações tecnológicas aliadas às ações pedagógicas, com uma estratégia condizente, é possível melhorar a qualidade de ensino e levar em consideração o perfil, as metas, as necessidades, as expectativas, as preferências e o nível de conhecimento de cada aluno, de modo a proporcionar a cada um, um ensino adaptado ( Falkembach, 2003 ). 
O resultado da utilização do A4 com recurso didático mostrou que a aprendizagem do conteúdo necessário ao desenvolvimento de um algoritmo foi potencializada, aumentando nos alunos a capacidade de abstrair e formalizar.

\section{O Ambiente A4}

O A4, Ambiente de Aprendizagem Adaptado para Algoritmos, é um sistema hipermídia interativo e utiliza a Estratégia Ascendente de Resolução de Problemas "[...] que é um plano de ação que determina que os itens, de um conteúdo, devem ser abordados passo a passo."( Falkembach, 2003 ). O termo adaptado equivale às adequações geradas pelas escolhas do próprio aluno. Significa adequação ao ritmo, à escolha da forma de apresentação do conteúdo e às preferências do aluno com relação a cores, tamanho da fonte, layout da interface de navegação, além da opção de ajuda para explicações adicionais e o acesso ao mapa do ambiente. Um ambiente de aprendizagem adaptado por suas características estimula o aluno, propicia a exploração de um conteúdo, consolida o que o aluno já aprendeu e instiga-o a continuar explorando, vencendo um módulo após o outro.

A primeira versão foi desenvolvida utilizando a Linguagem ASP (Active Server Pages), Java Script, Action Script e oferecia opções que se adaptavam às preferências do aluno. O ambiente, nessa versão, utilizava uma técnica de Cookies para guardar as informações dos alunos junto com as suas preferências, na máquina cliente.

Após o uso desta primeira versão, constatou-se algumas limitações e problemas de interface no ambiente, então a versão original foi reestruturada para agregar mais recursos e melhorar o sistema que já tinha sido implementado. Foram adicionados módulos para auxiliar o aluno e o professor como: cadastro de material didático; fórum sala de discussão entre outros recursos que possibilitaram uma maior interação entre o aluno e professor.

Esta nova versão do ambiente A4 foi implementada em Software Livre, ou seja, seu código fonte é aberto e qualquer usuário pode executar, copiar, distribuir, estudar, modificar e aperfeiçoar o ambiente. Foi desenvolvido utilizando as seguintes tecnologias: Banco de Dados MySQL, Linguagem JSP (Java Server Pages) e Java Script ( Araujo et. al, 2003).

\section{Objetivo do A4}

Potencializar a aprendizagem de Algoritmos através da Estratégia Ascendente, utilizada no ambiente, para trabalhar de forma mais eficiente os processos cognitivos referentes à Resolução de Problemas, em especial a abstração e a formalização dos procedimentos, necessários ao desenvolvimento de um algoritmo, fazendo com que o aluno visualize com maior clareza os procedimentos necessários à resolução de um problema.

\section{Funcionamento do Ambiente A4}

O A4 funciona como um recurso didático. É um ambiente que permite ao aluno estudar sozinho, tornando-o independente e capaz de buscar a resposta de suas dúvidas. No ambiente está o conteúdo básico de Algoritmos e exemplos que partem do abstrato para o concreto, permitindo ao aluno visualizar e compreender as operações feitas pelo computador ao executar um programa. O aluno digita os valores relacionados às 
variáveis de entrada e acompanha o processamento que mostra os valores intermediários das variáveis na memória. Com isso o aluno visualiza o que ocorre internamente quando a máquina está processando as instruções de um programa. A simulação dos exemplos diminui as dificuldades em abstrair e formalizar.

Paralelamente ao conteúdo de Algoritmos, é oferecido o conteúdo básico da linguagem Pascal, normalmente a primeira linguagem de programação trabalhada nos cursos da área das Ciências Exatas.

\subsection{Navegação do Ambiente A4}

A navegação no ambiente A4 é feita via um menu principal, em que cada opção disponibiliza as opções secundárias.

A estrutura de navegação do A4 é um roteiro guiado baseado na Estratégia Ascendente de Resolução de Problemas que permite aos iniciantes acessar o conteúdo da disciplina na ordem mais indicada para aqueles que estão trabalhando pela primeira vez com Algoritmos. No caso dos mais experientes, o conteúdo pode ser acessado segundo a vontade do aprendiz

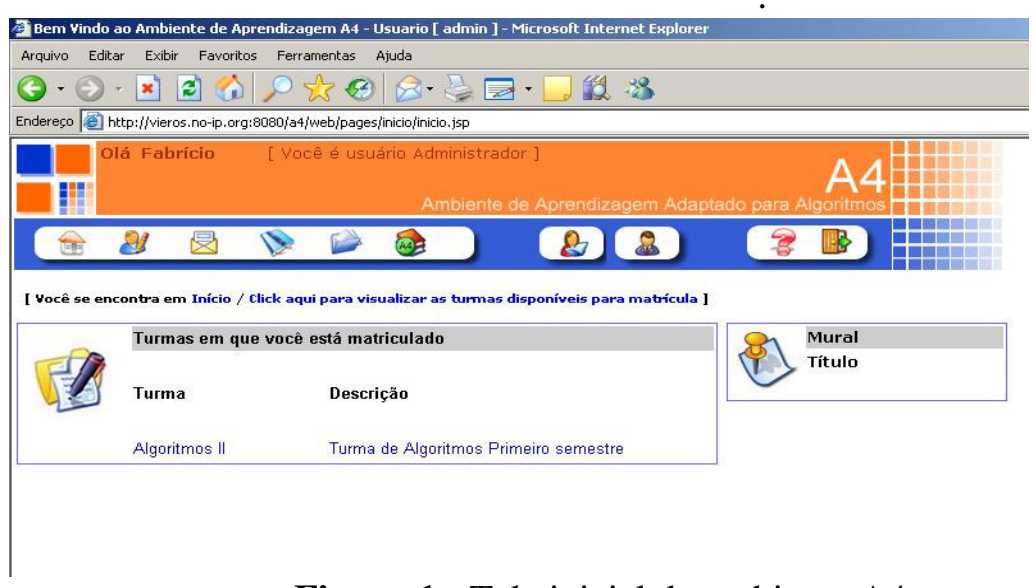

Figura 1 - Tela inicial do ambiente A4

\subsection{Problemas-Exemplo Interativos}

O ambiente A4 possui problemas-exemplo interativos elaborados nos modos de visualização disponíveis no ambiente que são: modo algoritmo, modo programa fonte e fluxograma como mostra a figura2. O modo programa fonte apresenta as instruções na linguagem de programação Pascal.

O aluno digita os valores das variáveis de entrada e o fluxo de processamento é mostrado no algoritmo, exibindo a alteração dos valores das variáveis na memória do computador. A simulação permite que o usuário digite os dados de entrada, acompanhe o processamento e os valores intermediários das variáveis na memória. Com isso o aluno segue a linha de raciocínio usada na elaboração do algoritmo para solucionar o problema. 


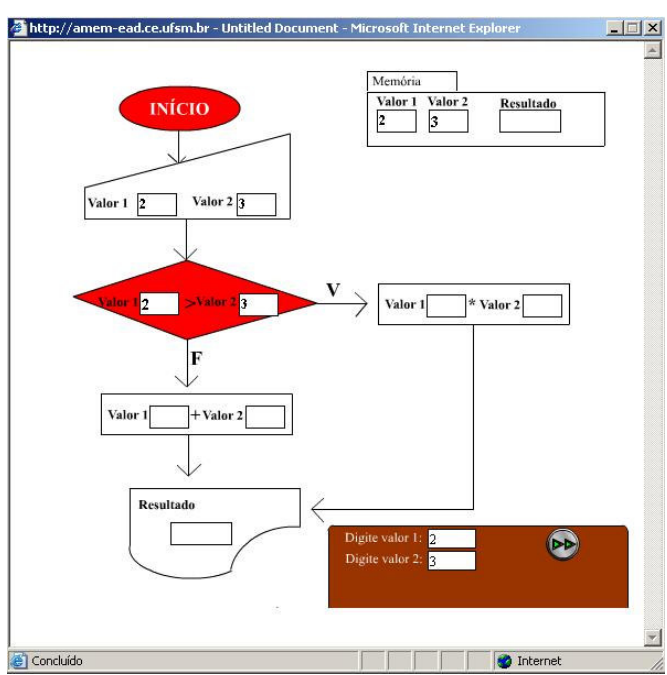

Figura 2 - Exemplo interativo de visualização de um algoritmo em fluxograma

\subsection{Módulos de Suporte ao Aluno e ao Professor}

A partir dos resultados satisfatórios obtidos com o uso do A4, como suporte ao ensino de Algoritmos, na versão original, foi feita a realimentação do ambiente e a implementação de alguns itens, gerando alterações no protótipo. Foi criado um sistema de gerenciamento para o professor, ampliando assim o número de informações a serem adicionadas no ambiente, como mostra a figura 4. Com os novos módulos incorporados ao ambiente, a na nova versão, disponibiliza os itens abaixo:

- Sistema de log-in - que permite um controle maior sobre os alunos;

- Fórum de Discussão - que permite a troca de informações entre os alunos e, entre os alunos e o professor possibilitando que professor e aluno interajam mais facilmente;

- Sala de discussão - que permite uma troca de idéias entre os alunos e, entre os alunos e o professor;

- Portfólio - que permite ao aluno disponibilizar os trabalhos para serem avaliados pelo professor;

- Mural - que permite a troca de informações entre professor e alunos;

- Material de Apoio - que permite que o professor disponibilize aos alunos material de aula, exercícios, tutoriais entre outros. Isso faz com que o sistema agregue um maior número de informações e não fique simplesmente restrito a um conteúdo estático; 


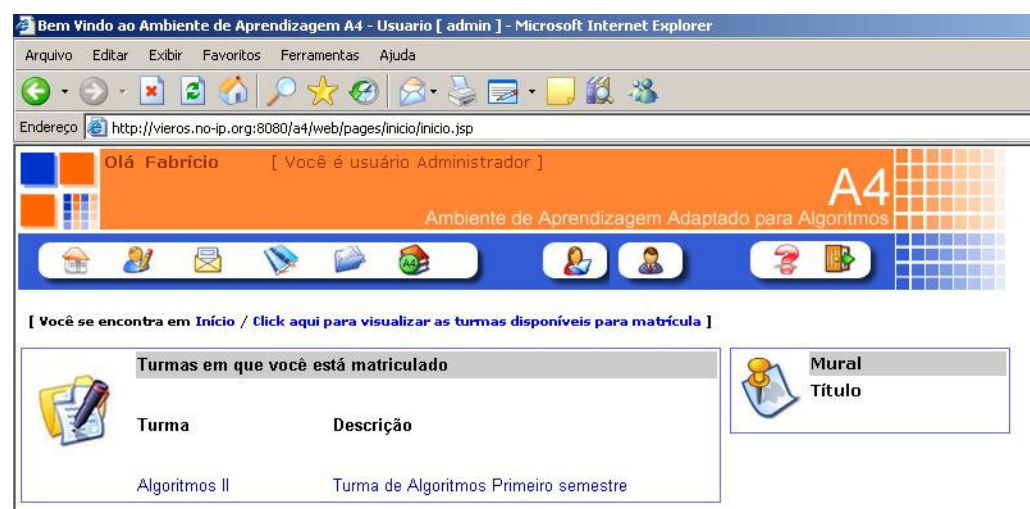

Figura 3 - Visão geral do Ambiente A4

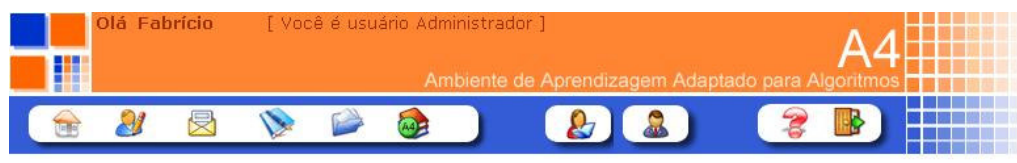

[ Você se encontra em Início / Gerenciamento do Professor ]

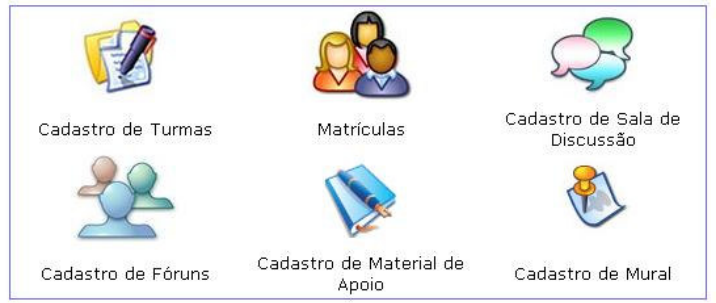

Figura 4 - Visão de gerenciamento do professor

\section{A Estratégia Ascendente para Resolução de Problemas}

Para desenvolver um algoritmo é preciso analisar as informações contidas no enunciado do problema, identificar o que é solicitado além de ter conhecimentos específicos que permitem planejar as ações para traçar a estratégia para a solução do problema. $\mathrm{O}$ conhecimento que contém a informação necessária ao desenvolvimento de um algoritmo se constitui em um novo elemento estratégico.

Existe uma grande variedade de estratégias. Um mesmo problema pode ser resolvido de várias maneiras, através de uma ou mais estratégias. Cada aluno adota uma estratégia, guiado por seu pensamento, como princípio de auto-instrução que auxilia no processo de resolução de problemas.

A estratégia ascendente é aquela cujos procedimentos são construídos passo a passo até chegar ao todo, ao resultado. A estratégia ascendente utiliza o raciocínio indutivo, sem confundir-se com ele. A estratégia ascendente envolve a subjetividade do 
aluno no desenvolvimento de um algoritmo. "Subjetividade é a interação do sujeito com a tarefa a ser executada".( Hoc, 2000)

A habilidade na resolução de problemas e a performance na programação dependem de certas características do aluno. Normalmente a estratégia de ensino utilizada pelo professor ao desenvolver o conteúdo de Lógica de Programação não é igualmente efetiva para todos os alunos. Alguns alunos se beneficiam mais que outros pelas interações entre suas características e a estratégia usada. A estratégia ascendente é uma maneira adequada de desenvolver um conteúdo como o de Algoritmos, pois trabalha de forma gradativa e induz o aluno, ao ter que resolver um problema, a buscar subsídios em problemas similares já resolvidos.

\section{Experiências Obtidas com o Uso do Ambiente}

O ambiente A4 já foi utilizado em turmas de diferentes faculdades e diferentes níveis de cursos. As experiências, aplicadas com o uso do ambiente em cursos de graduação de nível superior, mostraram que o mesmo como recurso didático contribuiu de maneira significativa para a aprendizagem do conteúdo teórico e para a desenvoltura na criação de algoritmos, minimizando as dificuldades inerentes à disciplina.

Os dados mostraram que os alunos acharam interessante o uso do ambiente, e que através dos exemplos interativos, contidos no A4, houve a possibilidade de visualizarem o funcionamento da resolução de um problema com os dados fornecidos por eles mesmos, tornando assim a aprendizagem dos procedimentos básicos, para o desenvolvimento de algoritmos, mais atrativa.

Os resultados das perguntas abertas e das observações anotadas no decorrer do semestre mostraram que o ambiente se comportou de forma aceitável dentro do que foi aplicado. No entanto, a partir de uma análise mais apurada, verificou-se que o ambiente pode ser melhorado com a inclusão de mais exemplos interativos e com maior grau de dificuldade além da inclusão dos recursos de mais uma linguagem de programação (Araújo, 2004).

O ambiente também foi aplicado em um curso de nível técnico, em que o tempo de utilização da ferramenta foi muito curto, fazendo com que esse fator fosse salientado nas observações dos alunos na tentativa de justificar porque o sucesso não foi maior no aprendizado de Algoritmos.

As experiências indicaram que o ambiente funciona como mais um recurso didático à disposição do professor e do aluno para o aprendizado dos procedimentos básicos utilizados na resolução de qualquer problema. Como, quando usar e de que forma usar esse recurso depende da metodologia de ensino usada pelo professor. Neste caso específico, o professor após as explicações em sala de aula, utilizou o ambiente como mais um material teórico, onde o aluno podia aprender conforme o seu ritmo e com o uso dos exemplos interativos, era mostrado o funcionamento dos algoritmos aplicados em sala de aula.

Os procedimentos na solução de um determinado problema é uma questão de organização de idéias e da escolha adequada de estratégias para criar a solução de um problema. Isso exige muito trabalho por parte do aluno e uma orientação segura por parte do professor e o ambiente pode auxiliar esse processo.

$\mathrm{O}$ aprendizado de Algoritmos se resume em fazer com que o aluno trabalhe com os conceitos básicos de Resolução de Problemas via computador e incorpore os subsídios que sustentam as abstrações e o formalismo da representação simbólica exigida na área computacional. 


\section{Conclusão}

O Ambiente de Aprendizagem Adaptado para Algoritmos já comprovou a partir de experiências em sala de aula, que quando aplicado como ferramenta de suporte ao aprendizado de Algoritmos, possibilita que o aluno aprenda o conteúdo de forma gradativa, pois a Estratégia Ascendente de Resolução de Problemas, utilizada envolve a subjetividade, que é a interação do aluno com a tarefa a ser executada e potencializa a aprendizagem. Essa estratégia aplicada no ambiente, faz com que os alunos com maior dificuldade aprendam a partir de problemas simples até os mais complexos e possibilita aquele aluno que possui uma maior facilidade de aprendizado possa estudar exemplos mais elaborados fazendo com que este aprimore o seu conhecimento mais rapidamente.

A partir dos resultados obtidos se pode concluir que o A4 contribuiu de maneira significativa para a aprendizagem do conteúdo teórico e para a desenvoltura na criação de algoritmos, minimizando as dificuldades provenientes desse conteúdo. Deve-se salientar que em todas os estudos analisados, o ambiente foi utilizado como ferramenta de apoio ao professor fazendo com que o mesmo trocasse conhecimentos entre os alunos e estes com o professor, não abolindo a forma tradicional de se aplicar a disciplina de algoritmos.

\section{Referências}

FALKEMBACH, G. A. M. Uma experiência de resolução de problemas através da estratégia ascendente: Ambiente de Aprendizagem Adaptado para Algoritmos (A4). Tese de Doutorado. Porto Alegre: PGIE/UFRGS, 2003.

ARAUJO, F.V ; FALKEMBACH, G.A.M (2003). - "Ambiente de Aprendizagem Adaptado Para Algoritmos" - Anais da Terceira Mostra de Trabalhos em Informática na Educação - TISE - Universidade do Chile - Santiago.

HOC, J.M (2000). "Psychologie Cognitive de la planification Grenoble: PUG”.Traduzido por Maria Suzana M. Amoretti. Porto Alegre - Brasil.

ARAUJO, F.V ; FALKEMBACH, G.A.M (2004). "Resultados da comparação na aprendizagem de Algoritmos, através de um Ambiente Adaptado ao Aprendizado, entre um Curso Técnico e um curso de Graduação" - I Congresso Sul Catarinense de Computação - 2005 - Criciúma/SC - Brasil.

GOMES, A.J.(2001) - "Ambiente de suporte à aprendizagem de conceitos básicos de programação" - Centro de Informática e Sistemas da Universidade de Coimbra Instituto Superior de Engenharia de Coimbra, Coimbra, Portugal.

QUARTIERO, E. M.(1996) "As tecnologias da Informação e a Comunicação e a Educação". <www.inf.ufsc.br/sbc_ie/revista/nr4/063RE-quartiero.htm>. 